Article

\title{
Stability of the Apollonius Type Additive Functional Equation in Modular Spaces and Fuzzy Banach Spaces
}

\author{
Sang Og Kim ${ }^{1, * \mathbb{C} \text { and John Michael Rassias }}{ }^{2}$ \\ 1 School of Data Science, Hallym University, Chuncheon 24252, Korea \\ 2 Pedagogical Department of Education E.E., Mathematics and Informatics Section, National and Capodistrian \\ University of Athens, 4, Agamemnonos St., Aghia Paraskevi, 15342 Athens, Greece; jrassias@primedu.uoa.gr \\ * Correspondence: sokim@hallym.ac.kr
}

Received: 29 October 2019; Accepted: 15 November 2019; Published: 17 November 2019

check for updates

\begin{abstract}
In this work, we investigate the generalized Hyers-Ulam stability of the Apollonius type additive functional equation in modular spaces with or without $\Delta_{2}$-conditions. We study the same problem in fuzzy Banach spaces and $\beta$-homogeneous Banach spaces. We show the hyperstability of the functional equation associated with the Jordan triple product in fuzzy Banach algebras. The obtained results can be applied to differential and integral equations with kernels of non-power types.
\end{abstract}

Keywords: Hyers-Ulam stability; fuzzy stability; Apollonius type additive functional equation; modular space; $\Delta_{2}$-condition; fuzzy Banach space

MSC: 39B52; 46A80; 46S40; 26E50

\section{Introduction and Preliminaries}

The research on modulars and modular spaces was begun by Nakano [1] as generalizations of normed spaces. Since the 1950s, many prominent mathematicians like Luxemburg, Mazur, Musielak, and Orlicz [2-5] developed it extensively. Modulars and modular spaces have broad branches of applications, e.g., interpolation theory and Orlicz spaces.

We start by considering some basic relevant notions.

Definition 1. ([1]) Let $X$ be a vector space over a field $\mathbb{K}(\mathbb{R}$ or $\mathbb{C})$. A generalized function $\rho: X \rightarrow[0, \infty]$ is called a modular if for any $\alpha, \beta \in \mathbb{K}$ and $x, y \in X$,

(1) $\rho(x)=0$ if and only if $x=0$,

(2) $\rho(\alpha x)=\rho(x)$ for every $\alpha$ with $|\alpha|=1$,

(3) $\rho(\alpha x+\beta y) \leq \rho(x)+\rho(y)$ if $\alpha+\beta=1$ and $\alpha, \beta \geq 0$.

If the condition (3) is replaced with

(4) $\rho(\alpha x+\beta y) \leq \alpha^{s} \rho(x)+\beta^{s} \rho(y)$ if $\alpha^{s}+\beta^{s}=1$ and $\alpha, \beta \geq 0$ with an $s \in(0,1]$,

then $\rho$ is called an s-convex modular. We call 1-convex modulars as convex modulars.

A modular $\rho$ on $X$ generates a linear subspace $X_{\rho}$ of $X$ naturally defined by

$$
X_{\rho}=\left\{x \in X \mid \lim _{\lambda \rightarrow 0} \rho(\lambda x)=0\right\}
$$

$X_{\rho}$ is called a modular space. 
Definition 2. Let $X_{\rho}$ be a modular space and $\left\{x_{n}\right\}$ be a sequence in $X_{\rho}$.

(1) $\left\{x_{n}\right\}$ is $\rho$-convergent to a point $x \in X_{\rho}$ if $\rho\left(x_{n}-x\right) \rightarrow 0$ as $n \rightarrow \infty$. The point $x$ is called the $\rho$-limit of the sequence $\left\{x_{n}\right\}$.

(2) $\left\{x_{n}\right\}$ is called a $\rho$-Cauchy sequence if $\rho\left(x_{n}-x_{m}\right) \rightarrow 0$ as $n, m \rightarrow \infty$.

(3) $X_{\rho}$ is called $\rho$-complete if every $\rho$-Cauchy sequence in $X_{\rho}$ is $\rho$-convergent.

Remark 1. If $\rho$ is a convex modular and $0 \leq \lambda \leq 1$, we have $\rho(\lambda x) \leq \lambda \rho(x)$ for all $x \in X_{\rho}$. If $\rho$ is a convex modular, and $\lambda_{i} \geq 0, i=1,2, \ldots$ nand $\lambda_{1}+\lambda_{2}+\ldots+\lambda_{n} \leq 1$, then $\rho\left(\lambda_{1} x_{1}+\lambda_{2} x_{2}+\ldots+\lambda_{n} x_{n}\right) \leq \lambda_{1} \rho\left(x_{1}\right)+$ $\lambda_{2} \rho\left(x_{2}\right)+\ldots+\lambda_{n} \rho\left(x_{n}\right)$. If $\left\{x_{n}\right\}$ is $\rho$-convergent to $x$, then $\left\{\alpha x_{n}\right\}$ is $\rho$-convergent to $\alpha x$, where $0 \leq \alpha \leq 1$. But the $\rho$-convergence of a sequence $\left\{x_{n}\right\}$ to $x$ does not imply that $\left\{c x_{n}\right\}$ is $\rho$-convergent to $c x$ for scalars $c$ with $|c|>1$.

There are two notions that play important roles when we study modulars. A modular $\rho$ is said to have the Fatou property if $\rho(x) \leq \liminf _{n \rightarrow \infty} \rho\left(x_{n}\right)$ for every sequence $\left\{x_{n}\right\}$ that is $\rho$-convergent to $x . \rho$ is said to satisfy the $\Delta_{2}$-condition if there exists a constant $\tau \geq 0$ such that $\rho(2 x) \leq \tau \rho(x)$ for all $x \in X_{\rho}$.

Example 1. For a measure space $(\Omega, \Sigma, \mu)$, let $L^{0}(\mu)$ be the collection of all measurable functions on $\Omega$. Let

$$
L^{\phi}(\mu)=\left\{f \in L^{0}(\mu) \mid \int_{\Omega} \phi(|\lambda f(x)|) d \mu(x) \rightarrow 0 \text { as } \lambda \rightarrow 0\right\},
$$

where $\phi:[0, \infty) \rightarrow \mathbb{R}$ is assumed to be a continuous, positive, convex and nondecreasing function increasing to infinity with $\phi(0)=0$. We can take, e.g., $\phi(t)=e^{t^{2}}-1 . L^{\phi}(\mu)$ is called an Orlicz space. Define for $f \in L^{\phi}(\mu)$,

$$
\rho_{\phi}(f)=\int_{\Omega} \phi(|f|) d \mu
$$

Then $\rho_{\phi}$ is a complete modular.

The question of stability of a functional equation concerns the existence of an exact solution near to the function satisfying the equation approximately. In 1940, Ulam [6] raised the first stability problem. He proposed a question whether there exists an exact homomorphism near an approximate homomorphism. Hyers [7] gave an answer in Banach spaces. Since then, many authors have investigated the stability problems. We refer to [8-13] for more information on the stability of functional equations.

The equality

$$
\|z-x\|^{2}+\|z-y\|^{2}=\frac{1}{2}\|x-y\|^{2}+2\left\|z-\frac{x+y}{2}\right\|^{2}
$$

which is called the Apollonius identity, holds in inner product spaces. It motivated the following quadratic functional equation,

$$
Q(z-x)+Q(z-y)=\frac{1}{2} Q(x-y)+2 Q\left(z-\frac{x+y}{2}\right) .
$$

So, Equation (1) is called the quadratic functional equation of Apollonius type. Jun and Kim [14] initiated the investigation of this functional equation.

Park and Rassias [15] introduced the following functional equation:

$$
f(z-x)+f(z-y)=-\frac{1}{2} f(x+y)+2 f\left(z-\frac{x+y}{4}\right),
$$

called the Apollonius type additive functional equation and investigated homomorphisms in $C^{*}$-ternary algebras and $J B^{*}$-triples associated with (2). In addition, in [16], the authors studied Jordan mappings in $C^{*}$-ternary algebras and $J B^{*}$-triples associated with (2). 
When studying the stability of functional equations, many authors work in normed spaces. However, there exist a number of topological spaces that are not normable. The concept of modulars is wider than that of norms, so modulars have less properties than norms have, but they make more sense in many particular situations. Working in a modular space, many authors often assume that the modular satisfies the Fatou property or $\Delta_{2}$-condition or both (see, e.g., $[17,18]$ ).

Recently, many authors investigated the stability of various functional equations on modular spaces. We refer the readers to [19-26].

This paper consists of six sections. In Section 2, we show the stability of the following functional equation without any condition on the modular;

$$
2 f(z-x)+2 f(z-y)=-f(x+y)+4 f\left(z-\frac{x+y}{4}\right) .
$$

In Section 3, we prove the stability of Apollonius type additive functional Equation (2) in modular spaces under the condition that the modular fulfills the $\Delta_{2}$-condition but not necessarily Fatou property.

In Section 4 , we obtain a similar result for $\beta$-homogeneous Banach spaces.

In Section 5, we show the fuzzy stability of the functional Equation (2) in fuzzy Banach spaces by using a fixed point method.

In Section 6, we show the hyperstability of the functional Equation (2) associated with the Jordan triple product in fuzzy Banach algebras.

\section{Stability of (3) in Modular Spaces Without $\Delta_{2}$-Conditions}

This section is concerned with the stability of the functional Equation (3). Dividing the functional equation by 2, this equation is reduced to the Apollonius type additive functional equation (2). Note that in the following theorem, the convex modular $\rho$ is not assumed to satisfy any other condition.

Lemma 1. [15] Let $V$ and $X$ be linear spaces and $f: V \rightarrow X$ be a mapping such that

$$
f(z-x)+f(z-y)+\frac{1}{2} f(x+y)-2 f\left(z-\frac{x+y}{4}\right)=0
$$

for all $x, y, z \in V$. Then $f$ is additive.

Theorem 1. Let $V$ be a linear space, $\rho$ be a convex modular, and $X_{\rho}$ be a $\rho$-complete modular space. Let $\varphi$ : $V^{3} \rightarrow[0, \infty)$ be a function with

$$
\widehat{\varphi}(x, y, z):=\sum_{k=1}^{\infty} \frac{1}{4^{k}} \varphi\left(4^{k-1} x, 4^{k-1} y, 4^{k-1} z\right)<\infty
$$

for all $x, y, z \in V$. Assume that $f: V \rightarrow X_{\rho}$ is a mapping satisfying $f(0)=0$ and

$$
\rho\left(2 f(z-x)+2 f(z-y)+f(x+y)-4 f\left(z-\frac{x+y}{4}\right)\right) \leq \varphi(x, y, z)
$$

for all $x, y, z \in V$. Then there exists a unique additive mapping $T: V \rightarrow X_{\rho}$ such that

$$
\rho(f(x)-T(x)) \leq \widehat{\varphi}(2 x, 2 x, 2 x), \quad x \in V .
$$

The mapping $T$ is defined by $T(x)=\rho$ - limit $\frac{f\left(4^{n} x\right)}{4^{n}}, \quad x \in V$.

Proof. Replacing $(x, y, z)$ with $(2 x, 2 x, 2 x)$ in (5) and letting $\psi(x)=\varphi(2 x, 2 x, 2 x)$, we have

$$
\rho(f(4 x)-4 f(x)) \leq \varphi(2 x, 2 x, 2 x)=\psi(x), \quad x \in V,
$$


hence,

$$
\rho\left(\frac{1}{4} f(4 x)-f(x)\right) \leq \frac{1}{4} \psi(x) .
$$

Then by induction, we write

$$
\rho\left(\frac{f\left(4^{k} x\right)}{4^{k}}-f(x)\right) \leq \sum_{j=1}^{k} \frac{1}{4^{j}} \psi\left(4^{j-1} x\right)
$$

for all $x \in V$ and all positive integer $k$. Indeed, the case $k=1$ follows from (8). Assume that (9) holds for $k \in \mathbb{N}$. Then we have the following inequality:

$$
\begin{aligned}
\rho\left(\frac{f\left(4^{k+1} x\right)}{4^{k+1}}-f(x)\right) & =\rho\left(\frac{1}{4}\left(\frac{f\left(4^{k} \cdot 4 x\right)}{4^{k}}-f(4 x)\right)+\frac{1}{4}(f(4 x)-4 f(x))\right) \\
& \leq \frac{1}{4} \rho\left(\frac{f\left(4^{k} \cdot 4 x\right)}{4^{k}}-f(4 x)\right)+\frac{1}{4} \rho(f(4 x)-4 f(x)) \\
& \leq \frac{1}{4} \sum_{j=1}^{k} \frac{1}{4^{j}} \psi\left(4^{j} x\right)+\frac{1}{4} \psi(x) \\
& =\sum_{j=1}^{k} \frac{1}{4^{j+1}} \psi\left(4^{j} x\right)+\frac{1}{4} \psi(x) \\
& =\sum_{j=1}^{k+1} \frac{1}{4^{j}} \psi\left(4^{j-1} x\right) .
\end{aligned}
$$

Hence, (9) holds for every $k \in \mathbb{N}$.

Let $m$ and $n$ be nonnegative integers with $n>m$. By (9), we have

$$
\begin{aligned}
\rho\left(\frac{f\left(4^{n} x\right)}{4^{n}}-\frac{f\left(4^{m} x\right)}{4^{m}}\right) & =\rho\left(\frac{1}{4^{m}}\left(\frac{f\left(4^{n-m} \cdot 4^{m} x\right)}{4^{n-m}}-f\left(4^{m} x\right)\right)\right) \\
& \leq \frac{1}{4^{m}} \sum_{j=1}^{n-m} \frac{\psi\left(4^{j-1} \cdot 4^{m} x\right)}{4^{j}} \\
& =\sum_{j=1}^{n-m} \frac{\psi\left(4^{m+j-1} x\right)}{4^{m+j}} \\
& =\sum_{k=m+1}^{n} \frac{\psi\left(4^{k-1} x\right)}{4^{k}} .
\end{aligned}
$$

Then (4) and (10) yield that $\left\{\frac{f\left(4^{n} x\right)}{4^{n}}\right\}$ is a $\rho$-Cauchy sequence in $X_{\rho}$. The $\rho$-completeness of $X_{\rho}$ guarantees its $\rho$-convergence. Hence, there exists a mapping $T: V \rightarrow X_{\rho}$ defined by

$$
T(x)=\rho-\operatorname{limit} \frac{f\left(4^{n} x\right)}{4^{n}}, \quad x \in V .
$$

We see that

$$
\begin{aligned}
\rho\left(\frac{T(4 x)-4 T(x)}{4^{3}}\right) & =\rho\left(\frac{1}{4^{3}}\left(T(4 x)-\frac{f\left(4^{n+1} x\right)}{4^{n}}\right)+\frac{1}{4}\left(\frac{1}{4} \cdot \frac{f\left(4^{n+1} x\right)}{4^{n+1}}-\frac{1}{4} T(x)\right)\right) \\
& \leq \frac{1}{4^{3}} \rho\left(T(4 x)-\frac{f\left(4^{n+1} x\right)}{4^{n}}\right)+\frac{1}{16} \rho\left(\frac{f\left(4^{n+1} x\right)}{4^{n+1}}-T(x)\right)
\end{aligned}
$$


for all $x \in V$. Then by (11), the right hand side of (12) tends to 0 as $n \rightarrow \infty$. Therefore, it follows that

$$
T(4 x)=4 T(x), \quad x \in V .
$$

Next, we calculate $\rho(T(x)-f(x))$. Note that for every $n \in \mathbb{N}$, by (13) we write

$$
\begin{aligned}
& \rho(T(x)-f(x)) \\
& =\rho\left(\sum_{k=1}^{n} \frac{f\left(4^{k} x\right)-4 f\left(4^{k-1} x\right)}{4^{k}}+\left(T(x)-\frac{f\left(4^{n} x\right)}{4^{n}}\right)\right) \\
& =\rho\left(\sum_{k=1}^{n} \frac{f\left(4^{k} x\right)-4 f\left(4^{k-1} x\right)}{4^{k}}+\frac{1}{4}\left(T(4 x)-\frac{f\left(4^{n-1} \cdot 4 x\right)}{4^{n-1}}\right)\right) .
\end{aligned}
$$

Since $\sum_{k=1}^{n} \frac{1}{4^{k}}+\frac{1}{4}<1$, it follows from (7) and (14) that

$$
\begin{aligned}
& \rho(T(x)-f(x)) \\
& \leq \sum_{k=1}^{n} \frac{1}{4^{k}} \rho\left(f\left(4^{k} x\right)-4 f\left(4^{k-1} x\right)\right)+\frac{1}{4} \rho\left(T(4 x)-\frac{f\left(4^{n-1} \cdot 4 x\right)}{4^{n-1}}\right) \\
& \leq \sum_{k=1}^{n} \frac{1}{4^{k}} \psi\left(4^{k-1} x\right)+\frac{1}{4} \rho\left(T(4 x)-\frac{f\left(4^{n-1} \cdot 4 x\right)}{4^{n-1}}\right) \\
& =\sum_{k=1}^{n} \frac{1}{4^{k}} \varphi\left(4^{k-1} \cdot 2 x, 4^{k-1} \cdot 2 x, 4^{k-1} \cdot 2 x\right)+\frac{1}{4} \rho\left(T(4 x)-\frac{f\left(4^{n-1} \cdot 4 x\right)}{4^{n-1}}\right), \quad x \in V .
\end{aligned}
$$

Letting $n \rightarrow \infty$ in (15), we obtain

$$
\rho(T(x)-f(x)) \leq \widehat{\varphi}(2 x, 2 x, 2 x), \quad x \in V .
$$

Therefore, we arrive at (6).

Now, we prove that $T$ is additive. We note that

$$
\begin{aligned}
& \rho\left(\frac{2 f\left(4^{j}(z-x)\right)}{4^{j}}+\frac{2 f\left(4^{j}(z-y)\right)}{4^{j}}+\frac{f\left(4^{j}(x+y)\right)}{4^{j}}-\frac{4 f\left(4^{j}\left(z-\frac{x+y}{4}\right)\right)}{4^{j}}\right) \\
& \leq \frac{1}{4^{j}} \rho\left(2 f\left(4^{j}(z-x)\right)+2 f\left(4^{j}(z-y)\right)+f\left(4^{j}(x+y)\right)-4 f\left(4^{j}\left(z-\frac{x+y}{4}\right)\right)\right) \\
& \leq \frac{1}{4^{j}} \varphi\left(4^{j} x, 4^{j} y, 4^{j} z\right) \rightarrow 0 \text { as } j \rightarrow \infty
\end{aligned}
$$

for all $x, y, z \in V$. 
Now, by (16) we have the following inequality

$$
\begin{aligned}
& \rho\left(\frac{1}{24}\left(2 T(z-x)+2 T(z-y)+T(x+y)-4 T\left(z-\frac{x+y}{4}\right)\right)\right) \\
& \leq \frac{1}{12}\left[\rho\left(T(z-x)-\frac{f\left(4^{j}(z-x)\right)}{4^{j}}\right)+\rho\left(T(z-y)-\frac{f\left(4^{j}(z-y)\right)}{4^{j}}\right)\right. \\
& \left.\quad+\frac{1}{2} \rho\left(T(x+y)-\frac{f\left(4^{j}(x+y)\right)}{4^{j}}\right)+2 \rho\left(T\left(z-\frac{x+y}{4}\right)-\frac{f\left(4^{j}\left(z-\frac{x+y}{4}\right)\right)}{4^{j}}\right)\right] \\
& \quad+\frac{1}{24} \rho\left(\frac{2 f\left(4^{j}(z-x)\right)}{4^{j}}+\frac{2 f\left(4^{j}(z-y)\right)}{4^{j}}+\frac{f\left(4^{j}(x+y)\right)}{4^{j}}-\frac{4 f\left(4^{j}\left(z-\frac{x+y}{4}\right)\right)}{4^{j}}\right) \\
& \rightarrow 0 \text { as } j \rightarrow \infty .
\end{aligned}
$$

Hence, we get

$$
T(z-x)+T(z-y)+\frac{1}{2} T(x+y)-2 T\left(z-\frac{x+y}{4}\right)=0
$$

for all $x, y, z \in V$. Then by Lemma 1 , it follows that $T$ is an additive mapping.

Finally, to show the uniqueness of $T$, assume that $T_{1}$ and $T_{2}$ are additive mappings satisfying (6). Then we write

$$
\begin{aligned}
\rho\left(\frac{T_{1}(x)-T_{2}(x)}{2}\right) & =\rho\left(\frac{1}{2}\left(\frac{T_{1}\left(4^{k} x\right)}{4^{k}}-\frac{f\left(4^{k} x\right)}{4^{k}}\right)+\frac{1}{2}\left(\frac{f\left(4^{k} x\right)}{4^{k}}-\frac{T_{2}\left(4^{k} x\right)}{4^{k}}\right)\right) \\
& \leq \frac{1}{2} \rho\left(\frac{T_{1}\left(4^{k} x\right)}{4^{k}}-\frac{f\left(4^{k} x\right)}{4^{k}}\right)+\frac{1}{2} \rho\left(\frac{f\left(4^{k} x\right)}{4^{k}}-\frac{T_{2}\left(4^{k} x\right)}{4^{k}}\right) \\
& \leq \frac{1}{2} \cdot \frac{1}{4^{k}}\left\{\rho\left(T_{1}\left(4^{k} x\right)-f\left(4^{k} x\right)\right)+\rho\left(T_{2}\left(4^{k} x\right)-f\left(4^{k} x\right)\right)\right\} \\
& \leq \frac{1}{4^{k}} \widehat{\varphi}\left(4^{k} \cdot 2 x, 4^{k} \cdot 2 x, 4^{k} \cdot 2 x\right) \\
& =\sum_{l=k+1}^{\infty} \frac{1}{4^{l}} \varphi\left(4^{l-1} \cdot 2 x, 4^{l-1} \cdot 2 x, 4^{l-1} \cdot 2 x\right) \\
& \rightarrow 0 \text { as } k \rightarrow \infty .
\end{aligned}
$$

This implies that $T_{1}=T_{2}$.

Now, we have the classical Ulam stability of (3) by putting $\varphi \equiv \varepsilon>0$.

Corollary 1. Let $V$ be a linear space, $\rho$ be a convex modular and $X_{\rho}$ be a $\rho$-complete modular space. Assume $f$ : $V \rightarrow X_{\rho}$ is a mapping such that $f(0)=0$ and

$$
\rho\left(2 f(z-x)+2 f(z-y)+f(x+y)-4 f\left(z-\frac{x+y}{4}\right)\right) \leq \varepsilon
$$

for all $x, y, z \in V$. Then there exists a unique additive mapping $T: V \rightarrow X_{\rho}$ such that

$$
\rho(f(x)-T(x)) \leq \frac{\varepsilon}{3}, \quad x \in V .
$$


Corollary 2. Let $V$ be a normed linear space, $\rho$ be a convex modular and $X_{\rho}$ be a $\rho$-complete modular space. Let $\theta>0$ and $0<p<1$ be real numbers. Assume that $f: V \rightarrow X_{\rho}$ is a mapping satisfying

$$
\rho\left(2 f(z-x)+2 f(z-y)+f(x+y)-4 f\left(z-\frac{x+y}{4}\right)\right) \leq \theta\left(\|x\|^{p}+\|y\|^{p}+\|z\|^{p}\right)
$$

for all $x, y, z \in V$. Then there exists a unique additive mapping $T: V \rightarrow X_{\rho}$ such that

$$
\rho(f(x)-T(x)) \leq \frac{3 \cdot 2^{p} \theta}{4-4^{p}}\|x\|^{p}, \quad x \in V .
$$

\section{Stability of (2) in Modular Spaces with $\Delta_{2}$-Conditions}

We show the generalized Hyers-Ulam stability of Apollonius type additive functional equation from linear spaces to modular spaces.

Theorem 2. Let $V$ be a linear space, $\rho$ be a convex modular satisfying the $\Delta_{2}$-condition with $\tau$ and $X_{\rho}$ be a $\rho$-complete modular space. Let $\varphi: V^{3} \rightarrow[0, \infty)$ be a function with

$$
\widehat{\varphi}(x, y, z):=\sum_{k=1}^{\infty} \frac{1}{4^{k}} \varphi\left(4^{k-1} x, 4^{k-1} y, 4^{k-1} z\right)<\infty
$$

for all $x, y, z \in V$. Assume that $f: V \rightarrow X_{\rho}$ is a mapping satisfying $f(0)=0$ and

$$
\rho\left(f(z-x)+f(z-y)+\frac{1}{2} f(x+y)-2 f\left(z-\frac{x+y}{4}\right)\right) \leq \varphi(x, y, z)
$$

for all $x, y, z \in V$. Then there exists a unique additive mapping $T: V \rightarrow X_{\rho}$ such that

$$
\rho(f(x)-T(x)) \leq \tau \widehat{\varphi}(2 x, 2 x, 2 x), \quad x \in V .
$$

Proof. Since $\rho$ satisfies the $\Delta_{2}$-condition with $\tau$, (17) implies

$$
\rho\left(2 f(z-x)+2 f(z-y)+f(x+y)-4 f\left(z-\frac{x+y}{4}\right)\right) \leq \tau \cdot \varphi(x, y, z)
$$

for all $x, y, z \in V$. Then the conclusion is a direct consequence of Theorem 1 .

Putting $\varphi \equiv \varepsilon>0$ in Theorem 2, we have the following result on classical Ulam stability of the Apollonius type additive functional equation.

Corollary 3. Let $V$ be a linear space, $\rho$ be a convex modular satisfying the $\Delta_{2}$-condition with $\tau$ and $X_{\rho}$ be a $\rho$-complete modular space. Assume $f: V \rightarrow X_{\rho}$ is a mapping satisfying $f(0)=0$ and

$$
\rho\left(f(z-x)+f(z-y)+\frac{1}{2} f(x+y)-2 f\left(z-\frac{x+y}{4}\right)\right) \leq \varepsilon
$$

for all $x, y, z \in V$. Then there exists a unique additive mapping $T: V \rightarrow X_{\rho}$ such that

$$
\rho(f(x)-T(x)) \leq \frac{\tau \cdot \varepsilon}{3}, \quad x \in V .
$$

\section{Stability of (2) in $\beta$-homogeneous Spaces}

Definition 3. Let $X$ be a linear space over $\mathbb{C}$. An F-norm is a function $\|\cdot\|: X \rightarrow[0, \infty]$ such that

(1) $\|x\|=0$ if and only if $x=0$, 
(2) $\|\lambda x\|=\|x\|$ for every $x \in X$ and every $\lambda$ with $|\lambda|=1$,

(3) $\|x+y\| \leq\|x\|+\|y\|$ for all $x, y \in X$,

(4) $\left\|\lambda_{n} x\right\| \rightarrow 0$ provided $\lambda_{n} \rightarrow 0$,

(5) $\left\|\lambda x_{n}\right\| \rightarrow 0$ provided $x_{n} \rightarrow 0$.

$(X, d)$ is a metric space by letting $d(x, y)=\|x-y\|$. It is called an F-space if $d$ is complete.

If, in addition, $\|t x\|=|t|^{\beta}\|x\|$ for all $x \in X$ and $t \in \mathbb{C}$, then $\|\cdot\|$ is called $\beta$-homogeneous $(\beta>0)$. A $\beta$-homogeneous $F$-space is called a $\beta$-homogeneous complex Banach space.

Remark 2. For an s-convex modular $\rho$, if we define

$$
\|x\|_{\rho}=\inf \left\{\alpha^{s}>0 \mid \rho\left(\frac{x}{\alpha}\right) \leq 1\right\}, \quad x \in X_{\rho}
$$

then $\|\cdot\|_{\rho}$ is an F-norm on $X_{\rho}$ such that $\|\lambda x\|_{\rho}=|\lambda|^{s}\|x\|_{\rho}$. Hence, $\|\cdot\|_{\rho}$ is s-homogeneous. For $s=1$, this norm is called the Luxemburg norm.

Considering Remark 2, we prove the generalized Hyers-Ulam stability of (2) from linear spaces to $\beta$-homogeneous Banach spaces.

Theorem 3. Let $V$ be a linear space, $X$ be a $\beta$-homogeneous complex Banach space $(0<\beta \leq 1)$, and $\varphi: V^{3} \rightarrow$ $[0, \infty)$ be a function with

$$
\widehat{\varphi}(x, y, z):=\frac{1}{2^{\beta}} \sum_{j=1}^{\infty} \frac{1}{4^{(j-1) \beta}} \varphi\left(4^{j-1} x, 4^{j-1} y, 4^{j-1} z\right)<\infty
$$

for all $x, y, z \in V$. Assume that $f: V \rightarrow X$ is a mapping satisfying $f(0)=0$ and

$$
\left\|f(z-x)+f(z-y)+\frac{1}{2} f(x+y)-2 f\left(z-\frac{x+y}{4}\right)\right\| \leq \varphi(x, y, z)
$$

for all $x, y, z \in V$. Then there exists a unique additive mapping $T: V \rightarrow X$ such that

$$
\|f(x)-T(x)\| \leq \widehat{\varphi}(2 x, 2 x, 2 x), \quad x \in V .
$$

Proof. Replacing $(x, y, z)$ with $(2 x, 2 x, 2 x)$ in (19), we get

$$
\left\|\frac{1}{2} f(4 x)-2 f(x)\right\| \leq \varphi(2 x, 2 x, 2 x), \quad x \in V .
$$

By induction on $k \in \mathbb{N}$, using (21) and putting $\psi(x)=\varphi(2 x, 2 x, 2 x)$, it is easy to see that

$$
\left\|\frac{f\left(4^{k} x\right)}{4^{k}}-f(x)\right\| \leq \frac{1}{2^{\beta}} \sum_{j=1}^{k} \frac{1}{4^{(j-1) \beta}} \psi\left(4^{j-1} x\right), \quad x \in V
$$


for all $k \in \mathbb{N}$. Let $m$ and $n$ be nonnegative integers with $n>m$. Then, by (22), we have

$$
\begin{aligned}
\left\|\frac{f\left(4^{n} x\right)}{4^{n}}-\frac{f\left(4^{m} x\right)}{4^{m}}\right\| & =\left\|\frac{1}{4^{m}}\left(\frac{f\left(4^{n} x\right)}{4^{n-m}}-f\left(4^{m} x\right)\right)\right\| \\
& \leq \frac{1}{4^{m \beta}} \cdot \frac{1}{2^{\beta}} \sum_{j=1}^{n-m} \frac{1}{4^{(j-1) \beta}} \psi\left(4^{j+m-1} x\right) \\
& \leq \frac{1}{2^{\beta}} \sum_{j=1}^{n-m} \frac{1}{4^{(j+m-1) \beta}} \psi\left(4^{j+m-1} x\right) \\
& =\frac{1}{2^{\beta}} \sum_{l=m+1}^{n} \frac{1}{4^{(l-1) \beta}} \psi\left(4^{l-1} x\right), \quad x \in V .
\end{aligned}
$$

Since the last expression of (23) goes to 0 by (18), it follows that, for every $x \in V$, the sequence $\left\{\frac{f\left(4^{n} x\right)}{4^{n}}\right\}$ is a Cauchy sequence in $X$. Since $X$ is complete, we know that the sequence is convergent. Hence, there exists a mapping $T: V \rightarrow X$ defined by

$$
T(x)=\lim _{n \rightarrow \infty} \frac{f\left(4^{n} x\right)}{4^{n}}, \quad x \in V .
$$

Letting $m=0$ and passing the limit $n \rightarrow \infty$ in (23), we obtain the estimate (20). In order to show that $T$ is additive, we write

$$
\begin{aligned}
& \left\|T(z-x)+T(z-y)+\frac{1}{2} T(x+y)-2 T\left(z-\frac{x+y}{4}\right)\right\| \\
& \leq\left\|T(z-x)-\frac{f\left(4^{j}(z-x)\right)}{4^{j}}\right\|+\left\|T(z-y)-\frac{f\left(4^{j}(z-y)\right)}{4^{j}}\right\| \\
& \quad+\frac{1}{2^{\beta}}\left\|T(x+y)-\frac{f\left(4^{j}(x+y)\right)}{4^{j}}\right\|+2^{\beta}\left\|T\left(z-\frac{x+y}{4}\right)-\frac{f\left(4^{j}\left(z-\frac{x+y}{4}\right)\right)}{4^{j}}\right\| \\
& \quad+\left\|\frac{f\left(4^{j}(z-x)\right)}{4^{j}}+\frac{f\left(4^{j}(z-y)\right)}{4^{j}}+\frac{f\left(4^{j}(x+y)\right)}{2 \cdot 4^{j}}-\frac{2 f\left(4^{j}\left(z-\frac{x+y}{4}\right)\right)}{4^{j}}\right\| \\
& \rightarrow 0 \text { as } j \rightarrow \infty .
\end{aligned}
$$

Hence, we get

$$
T(z-x)+T(z-y)+\frac{1}{2} T(x+y)-2 T\left(z-\frac{x+y}{4}\right)=0
$$

for all $x, y, z \in V$. Then by Lemma 1 , it follows that $T$ is an additive mapping.

Next, assume that $S: V \rightarrow X$ is another additive mapping satisfying (20). Then we have

$$
\begin{aligned}
& \|T(x)-S(x)\| \\
& \leq\left\|\frac{T\left(4^{k} x\right)-f\left(4^{k} x\right)}{4^{k}}\right\|+\left\|\frac{S\left(4^{k} x\right)-f\left(4^{k} x\right)}{4^{k}}\right\| \\
& \leq \frac{2}{4^{k \beta}} \frac{1}{2^{\beta}} \sum_{j=1}^{\infty} \frac{1}{4^{(j-1) \beta}} \varphi\left(2 \cdot 4^{k+j-1} x, 2 \cdot 4^{k+j-1} x, 2 \cdot 4^{k+j-1} x\right) \\
& =2^{1-\beta} \sum_{l=k+1}^{\infty} \frac{1}{4^{(l-1) \beta}} \varphi\left(2 \cdot 4^{l-1} x, 2 \cdot 4^{l-1} x, 2 \cdot 4^{l-1} x\right) \\
& \rightarrow 0 \text { as } k \rightarrow \infty
\end{aligned}
$$


for all $x \in V$, from which it follows that $T=S$.

Letting $\varphi \equiv \varepsilon>0$ in Theorem 3, we obtain a result on classical Ulam stability of the Apollonius type additive functional equation.

Corollary 4. Let $V$ be a linear space, and $X$ be a $\beta$-homogeneous complex Banach space with $0<\beta \leq 1$. If $f: V \rightarrow X$ is a mapping satisfying $f(0)=0$ and

$$
\left\|f(z-x)+f(z-y)+\frac{1}{2} f(x+y)-2 f\left(z-\frac{x+y}{4}\right)\right\| \leq \varepsilon
$$

for all $x, y, z \in V$, then there exists a unique additive mapping $T: V \rightarrow X$ such that

$$
\|f(x)-T(x)\| \leq \frac{2^{\beta} \epsilon}{4^{\beta}-1}, \quad x \in V .
$$

\section{Fuzzy Stability of (2) in Fuzzy Banach Spaces}

In this section we consider the stability of the Apollonius type additive functional Equation (2). The following theorem is a fundamental result in fixed point theory.

Theorem 4. $[27,28]$ Let $(X, d)$ be a complete generalized metric space and let $J: X \rightarrow X$ be a strictly contractive mapping with Lipschitz constant $L<1$. Then for each given element $x \in X$, either

$$
d\left(J^{n} x, J^{n+1} x\right)=\infty
$$

for all nonnegative integers $n$ or there exists a positive integer $n_{0}$ such that

1. $d\left(J^{n} x, J^{n+1} x\right)<\infty$, for all $n \geq n_{0}$;

2. the sequence $\left\{J^{n} x\right\}$ converges to a fixed point $y^{*}$ of $J$;

3. $y^{*}$ is the unique fixed point of $J$ in the set $Y=\left\{y \in X \mid d\left(J^{n_{0}} x, y\right)<\infty\right\}$;

4. $d\left(y, y^{*}\right) \leq \frac{1}{1-L} d(y, J y)$ for all $y \in Y$.

We use the definition of fuzzy normed spaces given in [29-31].

Definition 4. [29-31] Let $X$ be a real vector space. A function $N: X \times \mathbb{R} \rightarrow[0,1]$ is called a fuzzy norm on $X$ if for all $x, y \in X$ and all $s, t \in \mathbb{R}$,

(1) $N(x, t)=0$ for $t \leq 0$;

(2) $x=0$ if and only if $N(x, t)=1$ for all $t>0$;

(3) $N(c x, t)=N\left(x, \frac{t}{|c|}\right)$ if $c \neq 0$;

(4) $N(x+y, s+t) \geq \min \{N(x, s), N(y, t)\}$;

(5) $\quad N(x, \cdot)$ is a non-decreasing function of $\mathbb{R}$ and $\lim _{t \rightarrow \infty} N(x, t)=1$;

(6) for $x \neq 0, N(x, \cdot)$ is continuous on $\mathbb{R}$.

The pair $(X, N)$ is called a fuzzy normed vector space.

Example 2. Let $(X,\|\cdot\|)$ be a normed linear space. Then

$$
N(x, t)= \begin{cases}\frac{t}{t+\|x\|}, & x \in X, t>0 \\ 0, & x \in X, t \leq 0\end{cases}
$$

is a fuzzy norm on $X$.

Definition 5. [29-31] Let $(X, N)$ be a fuzzy normed vector space. A sequence $\left\{x_{n}\right\}$ in $X$ is said to be convergent to $x \in X$ if $\lim _{n \rightarrow \infty} N\left(x_{n}-x, t\right)=1$ for all $t>0$ and we denote it by $N$ - $\lim _{n \rightarrow \infty} x_{n}=x$. 
Definition 6. [29-31] Let $(X, N)$ be a fuzzy normed vector space. A sequence $\left\{x_{n}\right\}$ in $X$ is called a Cauchy sequence if $\lim _{n, m \rightarrow \infty} N\left(x_{n}-x_{m}, t\right)=1$ for all $t>0$.

If each Cauchy sequence is convergent, then the fuzzy norm is said to be complete, and the fuzzy normed vector space is called a fuzzy Banach space.

Definition 7. [32] Let $X$ be an algebra and $(X, N)$ a fuzzy Banach space. Then the space $(X, N)$ is said to be a fuzzy Banach algebra if

$$
N(x y, a b) \geq N(x, a) \circ N(y, b), x, y \in X, a, b \in \mathbb{R},
$$

where $\circ$ is a continuous t-norm.

Example 3. Let $X$ be an algebra and $(X, N)$ be a fuzzy Banach space, where $N(x, t)$ is given as in Example 2. Then

$$
N(x y, a b) \geq N(x, a) \cdot N(y, b), x, y \in X, a, b \in \mathbb{R} .
$$

Hence, $(X, N)$ is a fuzzy Banach algebra with $\circ=\cdot$, the product, (see [32]).

Let $(X, N)$ be a fuzzy Banach algebra and $x_{n} \rightarrow x$ and $y_{n} \rightarrow y$ be convergent sequences in $(X, N)$. It is easy to show that $N-\lim x_{n} y_{n}=x y$ (see [32]).

Now, we show the generalized Hyers-Ulam stability of (2) in fuzzy normed vector spaces via a fixed point method.

Theorem 5. Let $X$ be a real vector space, $(Y, N)$ a fuzzy Banach space. Let $\varphi: X^{3} \rightarrow[0, \infty)$ be a function such that $\varphi(0,0,0)=0$ and there exists an $0<L<1$ satisfying

$$
\varphi(4 x, 4 y, 4 z) \leq 4 L \varphi(x, y, z)
$$

for all $x, y, z \in X$. Let $f: X \rightarrow Y$ be a mapping that satisfies

$$
N\left(f(z-x)+f(z-y)+\frac{1}{2} f(x+y)-2 f\left(z-\frac{x+y}{4}\right), t\right) \geq \frac{t}{t+\varphi(x, y, z)}
$$

for all $x, y, z \in X, t>0$. Then there exists a unique additive mapping $F: X \rightarrow Y$ such that

$$
N(f(x)-F(x), t) \geq \frac{4(1-L) t}{4(1-L) t+2 \varphi(2 x, 2 x, 2 x)}, \quad x \in X, t>0 .
$$

The mapping $F$ is defined by $F(x)=N-\lim _{n \rightarrow \infty} \frac{1}{4^{n}} f\left(4^{n} x\right), \quad x \in X$.

Proof. Replacing $(x, y, z)$ with $(0,0,0)$ in $(26)$, we get

$$
N\left(\frac{1}{2} f(0), t\right) \geq \frac{t}{t+\varphi(0,0,0)}=1, \quad t>0,
$$

so that $f(0)=0$.

Replacing $(x, y, z)$ with $(2 x, 2 x, 2 x)$, we see

$$
N\left(\frac{1}{2} f(4 x)-2 f(x), t\right) \geq \frac{t}{t+\varphi(2 x, 2 x, 2 x)} .
$$

Then, putting

$$
M(x, t):=\frac{t}{t+2 \varphi(2 x, 2 x, 2 x)}, \quad x \in X, t>0
$$


we have

$$
N(f(4 x)-4 f(x), 2 t)=N\left(\frac{1}{2} f(4 x)-2 f(x), t\right) \geq \frac{t}{t+\varphi(2 x, 2 x, 2 x)}
$$

hence,

$$
N(f(4 x)-4 f(x), t) \geq \frac{t}{t+2 \varphi(2 x, 2 x, 2 x)}=M(x, t), \quad x \in X, t>0 .
$$

By the definition of $M(x, t)$ and (25), we note that for every $n \in \mathbb{N}$,

$$
\left.M\left(4^{n} x, 4^{n} L^{n} t\right)\right) \geq M(x, t), \quad x \in X, t>0 .
$$

Consider the set

$$
S=\{p \mid p: X \rightarrow Y\}
$$

and introduce the generalized metric on $S$ by

$$
d(p, q)=\inf \left\{\mu \in \mathbb{R}^{+} \mid N(p(x)-q(x), \mu t) \geq M(x, t), \quad x \in X, t>0\right\} .
$$

Then $(S, d)$ is a complete generalized metric space (see [33], Lemma 2.1). Now, we consider the map $J: S \rightarrow S$ given by

$$
J p(x)=\frac{1}{4} p(4 x), \quad x \in X
$$

Then by a standard argument, we know that $J$ is a contractive mapping.

Since $N(f(4 x)-4 f(x), t) \geq M(x, t)$ by (28), we have

$$
N\left(\frac{1}{4} f(4 x)-f(x), \frac{1}{4} t\right) \geq M(x, t), \quad x \in X, t>0 .
$$

Hence, it follows that

$$
d(J f, f) \leq \frac{1}{4}
$$

Then by the fixed point alternative, i.e., Theorem 4, there exists a mapping $F: X \rightarrow Y$ such that

1. $F$ is a fixed point of $J$, i.e.,

$$
F(4 x)=4 F(x), \quad x \in X
$$

2. $\quad d\left(J^{n} f, F\right) \rightarrow 0$, i.e.,

$$
N-\lim _{n \rightarrow \infty} \frac{1}{4^{n}} f\left(4^{n} x\right)=F(x), \quad x \in X .
$$

3. $\quad d(f, F) \leq \frac{1}{1-L} d(f, J f)$, i.e.,

$$
d(f, F) \leq \frac{1}{4(1-L)}
$$

This implies

$$
N(f(x)-F(x), t) \geq M(x, 4(1-L) t), \quad x \in X, t>0 .
$$

Note that (29) shows the inequality (27).

Now, we show that $F$ is an additive mapping. 
Replacing $(x, y, z)$ with $\left(4^{n} x, 4^{n} y, 4^{n} z\right)$ in (26), we get

$$
\begin{aligned}
& N\left(\frac{1}{4^{n}} f\left(4^{n}(z-x)\right)+\frac{1}{4^{n}} f\left(4^{n}(z-y)\right)+\frac{1}{2 \cdot 4^{n}} f\left(4^{n}(x+y)\right)-2 \cdot \frac{1}{4^{n}} f\left(4^{n} z-\frac{1}{4}\left(4^{n}(x+y)\right)\right), t\right) \\
& \geq \frac{4^{n} t}{4^{n} t+\varphi\left(4^{n} x, 4^{n} y, 4^{n} z\right)} \\
& \geq \frac{t}{t+L^{n} \varphi(x, y, z)} .
\end{aligned}
$$

Hence, it follows that

$$
F(z-x)+F(z-y)+\frac{1}{2} F(x+y)-2 F\left(z-\frac{x+y}{4}\right)=0
$$

for all $x, y, z \in X$.

Then by Lemma 1 , it follows that $F$ is additive.

Finally, assume that $F_{1}$ and $F_{2}$ are two additive mappings that satisfy (27). Then

$$
N\left(f(x)-F_{i}(x), t\right) \geq M(x, 4(1-L) t), \quad x \in X, t>0, i=1,2 .
$$

Then, by (29), we have

$$
\begin{aligned}
N\left(F_{2}(x)-F_{1}(x), 2 t\right) & =N\left(\frac{1}{4^{n}}\left(F_{2}\left(4^{n} x\right)-F_{1}\left(4^{n} x\right)\right), 2 t\right) \\
& \geq \min \left\{N\left(\frac{1}{4^{n}}\left(f\left(4^{n} x\right)-F_{1}\left(4^{n} x\right)\right), t\right), N\left(\frac{1}{4^{n}}\left(f\left(4^{n} x\right)-F_{2}\left(4^{n} x\right)\right), t\right)\right\} \\
& \geq M\left(4^{n} x,(1-L) 4^{n+1} t\right) \geq \frac{(1-L) 4^{n+1} t}{(1-L) 4^{n+1} t+2 \cdot(4 L)^{n} \varphi(2 x, 2 x, 2 x)} \\
& \rightarrow 1 \text { as } n \rightarrow \infty .
\end{aligned}
$$

This yields that $F_{1}=F_{2}$, as desired.

Corollary 5. Let $X$ be a real normed linear space, and $(Y, N)$ be a fuzzy Banach space. Let $\theta>0$ and $0<r<1$ be real numbers. Let $f: X \rightarrow Y$ be a mapping satisfying

$$
N\left(f(z-x)+f(z-y)+\frac{1}{2} f(x+y)-2 f\left(z-\frac{x+y}{4}\right), t\right) \geq \frac{t}{t+\theta\left(\|x\|^{r}+\|y\|^{r}+\|z\|^{r}\right)}
$$

for $x, y, z \in X$ and $t>0$. Then there exists a unique additive mapping $F: X \rightarrow Y$ such that

$$
N(f(x)-F(x), t) \geq \frac{\left(1-4^{r-1}\right) t}{\left(1-4^{r-1}\right) t+3 \cdot 2^{r-1} \theta\|x\|^{r}}
$$

for $x \in X, t>0$.

Proof. Taking $\varphi(x, y, z)=\theta\left(\|x\|^{r}+\|y\|^{r}+\|z\|^{r}\right)$ from Theorem 5, we can choose $L=4^{r-1}$ to get the result.

\section{Hyperstability of (2) in Fuzzy Banach Algebras}

In this section, we consider the Apollonius type additive functional equation on fuzzy Banach algebras. Let $X$ be a real algebra. An additive mapping $D: X \rightarrow X$ is called a derivation if

$$
D(x y)=D(x) y+x D(y), \quad x, y \in X .
$$


An additive mapping $D: X \rightarrow X$ is called a Jordan derivation if

$$
D\left(x^{2}\right)=D(x) x+x D(x), \quad x \in X .
$$

In addition, an additive mapping $D: X \rightarrow X$ is called a Jordan triple derivation in the sense from [34] if

$$
D(x y x)=D(x) y x+x D(y) x+x y D(x), \quad x, y \in X .
$$

It is well-known that every derivation is a Jordan derivation and every Jordan derivation is a Jordan triple derivation. However, the converse implications do not hold in general.

Theorem 6. Let $(X, N)$ be a fuzzy Banach algebra. Let $\varphi: X^{3} \rightarrow[0, \infty)$ be a function such that $\varphi(0,0,0)=0$ and there exists an $0<L<1$ satisfying

$$
\varphi(4 x, 4 y, 4 z) \leq 4 L \varphi(x, y, z)
$$

for all $x, y, z \in X$. Assume $f: X \rightarrow X$ is a mapping that satisfies

$$
\begin{aligned}
& \text { (a) } N\left(f(z-x)+f(z-y)+\frac{1}{2} f(x+y)-2 f\left(z-\frac{x+y}{4}\right), t\right) \geq \frac{t}{t+\varphi(x, y, z)}, \\
& \text { (b) } N(f(x y x)-f(x) y x-x f(y) x-x y f(x), t) \geq \frac{t}{t+\varphi(x, y, 0)}
\end{aligned}
$$

for all $x, y, z \in X, t>0$. Then there exists a unique Jordan triple derivation $F: X \rightarrow X$ such that

$$
N(f(x)-F(x), t) \geq \frac{4(1-L) t}{4(1-L) t+2 \varphi(2 x, 2 x, 2 x)}, \quad x \in X, t>0 .
$$

The mapping $F$ is defined by $F(x)=N-\lim _{n \rightarrow \infty} \frac{1}{4^{n}} f\left(4^{n} x\right), \quad x \in X$.

Proof. By Theorem 5, the mapping $F$ is additive. Replacing $(x, y)$ with $\left(4^{n} x, 4^{n} y\right)$ in $(31)$, we have by (30)

$$
\begin{aligned}
& N\left(\frac{1}{4^{3 n}} f\left(4^{3 n} x y x\right)-\frac{1}{4^{3 n}} 4^{2 n} f\left(4^{n} x\right) y x-\frac{1}{4^{3 n}} 4^{2 n} x f\left(4^{n} y\right) x-\frac{1}{4^{3 n}} 4^{2 n} x y f\left(4^{n} x\right), t\right) \\
& =N\left(f\left(4^{3 n} x y x\right)-4^{2 n} f\left(4^{n} x\right) y x-4^{2 n} x f\left(4^{n} y\right) x-4^{2 n} x y f\left(4^{n} x\right), 4^{3 n} t\right) \\
& \geq \frac{4^{3 n} t}{4^{3 n} t+\varphi\left(4^{n} x, 4^{n} y, 0\right)} \\
& \geq \frac{4^{3 n} t}{4^{3 n} t+(4 L)^{n} \varphi(x, y, 0)} \\
& =\frac{t}{t+\left(\frac{L}{16}\right)^{n} \varphi(x, y, 0)}
\end{aligned}
$$

from which we infer that

$$
F(x y x)=F(x) y x+x F(y) x+x y F(x), \quad x, y \in X .
$$

Therefore, $F$ is a Jordan triple derivation.

An algebra $A$ is called semiprime if whenever $a A a=\{0\}$ for $a \in A$, then $a=0 . B(X)$ for Banach spaces $X$ and all $C^{*}$-algebras are examples of semiprime algebras. A ring $R$ is said to be 2-torsion free if $2 r=0$ implies $r=0$ for $r \in R$. 
In the following theorem, we show that the mapping $f$ in Theorem 6 is a derivation if the algebra is semiprime.

Theorem 7. Let $(X, N)$ be a unital 2-torsion free semiprime fuzzy Banach algebra. Let $\varphi: X^{3} \rightarrow[0, \infty)$ be a function such that $\varphi(0,0,0)=0$ and there exists an $0<L<1$ satisfying, for all $x, y, z \in X$,

$$
\begin{aligned}
& \text { (a) } \varphi(4 x, 4 y, 4 z) \leq 4 L \varphi(x, y, z) \\
& \text { (b) }\left\{\frac{1}{4^{n}} \varphi\left(x, \frac{y}{4^{n}}, 0\right) \mid n \in \mathbb{N}\right\} \text { is bounded. }
\end{aligned}
$$

Assume $f: X \rightarrow X$ is a mapping such that, for all $x, y, z \in X$ and $t>0$,

$$
\begin{aligned}
& \text { (c) } N\left(f(z-x)+f(z-y)+\frac{1}{2} f(x+y)-2 f\left(z-\frac{x+y}{4}\right), t\right) \geq \frac{t}{t+\varphi(x, y, z)}, \\
& \text { (d) } N(f(x y x)-f(x) y x-x f(y) x-x y f(x), t) \geq \frac{t}{t+\varphi(x, y, 0)} .
\end{aligned}
$$

Then $f$ is an additive derivation.

Proof. Recall that the mapping $F$ defined by $F(x)=N-\lim _{n \rightarrow \infty} \frac{1}{4^{n}} f\left(4^{n} x\right), x \in X$ from Theorem 6 is an additive Jordan triple derivation. Replacing $(x, y)$ with $\left(4^{n} x, y\right)$ in (34), we have by (33)

$$
\begin{aligned}
& N\left(\frac{1}{4^{2 n}} f\left(4^{2 n} x y x\right)-\frac{1}{4^{2 n}} 4^{n} f\left(4^{n} x\right) y x-\frac{1}{4^{2 n}} 4^{2 n} x f(y) x-\frac{1}{4^{2 n}} 4^{n} x y f\left(4^{n} x\right), t\right) \\
& =N\left(f\left(4^{2 n} x y x\right)-4^{n} f\left(4^{n} x\right) y x-4^{2 n} x f(y) x-4^{n} x y f\left(4^{n} x\right), 4^{2 n} t\right) \\
& \geq \frac{4^{2 n} t}{4^{2 n} t+\varphi\left(4^{n} x, y, 0\right)} \\
& \geq \frac{4^{2 n} t}{4^{2 n} t+(4 L)^{n} \varphi\left(x, \frac{y}{4^{n}}, 0\right)} \\
& =\frac{t}{t+\left(\frac{L}{4}\right)^{n} \varphi\left(x, \frac{y}{4^{n}}, 0\right)^{\prime}}
\end{aligned}
$$

from which we get

$$
F(x y x)=F(x) y x+x f(y) x+x y F(x)
$$

for all $x, y \in X$. Comparing (35) with (32), it follows that

$$
x F(y) x=x f(y) x
$$

for all $x, y \in X$. Letting $x=1$, we conclude that $F=f$. Therefore, $f$ is a Jordan triple derivation. By [34], (Theorem 4.3), every Jordan triple derivation on a 2-torsion free semiprime ring is a derivation. Hence, we conclude that $f$ is an additive derivation.

Now, we have an application of Theorem 7 to simple $C^{*}$-algebras.

Corollary 6. Let $X$ be a unital simple $C^{*}$-algebra, $\theta>0$ and $0<r<1$ be real numbers. Assume that $f: X \rightarrow X$ is a mapping such that

$$
\begin{gathered}
\left\|f(z-x)+f(z-y)+\frac{1}{2} f(x+y)-2 f\left(z-\frac{x+y}{4}\right)\right\| \leq \theta\left(\|x\|^{r}+\|y\|^{r}+\|z\|^{r}\right), \\
\|f(x y x)-f(x) y x-x f(y) x-x y f(x)\| \leq \theta\left(\|x\|^{r}+\|y\|^{r}\right)
\end{gathered}
$$


for all $x, y, z \in X$. Then there exists an element $a \in X$ such that

$$
f(x)=a x-x a, \quad x \in X
$$

Proof. Letting $L=4^{r-1}, N(x, t)$ be as in Example 2 and $\varphi(x, y, z)=\theta\left(\|x\|^{r}+\|y\|^{r}+\|z\|^{r}\right)$, we have that $f$ is a derivation by Theorem 7 . It is well-known that every derivation on simple $C^{*}$-algebras is inner. Hence, we get the result.

\section{Conclusions}

Using the direct method, we have proved the Hyers-Ulam stability of Apollonius type additive functional equation from linear spaces to modular spaces with or without the $\Delta_{2}$-conditions. Since spaces with $s$-convex modulars are $s$-homogeneous Banach spaces, we also have investigated the same problem for $\beta$-homogeneous Banach spaces. The obtained results can be applied to normed spaces as well. We also have shown the fuzzy stability of the functional equation in fuzzy Banach spaces by using a fixed point method. Finally, we have shown the hyperstability of the functional Equation (2) associated with the Jordan triple product in fuzzy Banach algebras. Removing the $\Delta_{2}$-condition in Theorem 2 will be a challenging problem.

Author Contributions: The authors contributed equally to this work.

Funding: This work was supported by Hallym University Research Fund, 2019 (HRF-201909-017).

Acknowledgments: The authors are very grateful to the anonymous reviewers for their valuable suggestions which helped improving this paper.

Conflicts of Interest: The authors declare no conflict of interest.

\section{References}

1. Nakano, H. Modulared Semi-Ordered Linear Spaces; Maruzen: Tokyo, Japan, 1950.

2. Luxemburg, W.A.J. Banach Function Spaces. Ph.D. Thesis, Delft University of Technology, Delft, The Netherlands, 1955.

3. Mazur, S.; Orlicz, W. On some classes of linear spaces. Studia Math. 1958, 17, 97-119. [CrossRef]

4. Musielak, J.; Orlicz, W. On modular spaces. Studia Math. 1959, 18, 591-597. [CrossRef]

5. Musielak, J.; Orlicz, W. Some remarks on modular spaces. Bull. Acad. Polon. Sci. Sr. Math. Astron. Phys. 1959, 7, 661-668.

6. Ulam, S.M. Problems of Modern Mathematics; Sciences Editions; John Wiley \& Sons Inc.: New York, NY, USA, 1964.

7. Hyers, D.H. On the stability of linear functional equations. Proc. Nat. Acad. Sci. USA 1941, 27, $222-224$. [CrossRef] [PubMed]

8. Brillouët-Bellout, N.; Brzdęk, J.; Ciepliński, K. On some recent developments in Ulam's type stability. Abstr. Appl. Anal. 2012, 2012, 716936. [CrossRef]

9. Czerwik, S. Functional Equations and Inequalities in Several Variables; World Scientific: River Edge, NJ, USA, 2002.

10. Găvruta, P. A generalization of the Hyers-Ulam-Rassias stability of approximate additive mappings. J. Math. Anal. Appl. 1994, 184, 431-436. [CrossRef]

11. Hyers, D.H.; Isac, G.; Rassias, T.M. Stability of Functional Equations in Several Variables; Birkhäuser: Basel, Switzerland, 1998.

12. Jung, S. Hyers-Ulam-Rassias Stability of Functional Equations in Mathematical Analysis; Springer: New York, NY, USA, 2011.

13. Rassias, T.M. On the stability of linear mappings in Banach spaces. Proc. Amer. Math. Soc. 1978, 72, $297-300$. [CrossRef]

14. Jun, K.; Kim, H. On the stability of Apollonius' equation. Bull. Belg. Math. Soc.-Simon Stevin 2004, 11, 615-624.

15. Park, C.; Rassias, T.M. Homomorphisms in $C^{*}$-ternary algebras and JB*-triples. J. Math. Anal. Appl. 2008, 337, 13-20. [CrossRef]

16. Moghadam, M.R.; Rassias, T.M.; Keshavarz, V.; Park, C.; Park, Y.S. Jordan homomorphisms in $C^{*}$-ternary algebras and JB*-triples. J. Comput. Anal. Appl. 2018, 24, 416-424.

17. Khamsi, M.A. Quasicontraction mappings in modular spaces without $\Delta_{2}$-condition. Fixed Point Theory Appl. 2008, 2008, 916187. [CrossRef]

18. Khamsi, M.A.; Kozlowski, W.M. Fixed Point Theory in Modular Function Spaces; Birkhäuser: Basel, Switzerland, 2015. 
19. Cho, Y.J.; Ghaemi, M.B.; Choubin, M.; Gordji, M.E. On the Hyers-Ulam stability of sextic functional equations in $\beta$-homogeneous probabilistic modular spaces. Math. Inequal. Appl. 2013, 16, 1097-1114. [CrossRef]

20. Gordji, M.E.; Sajadian, F.; Cho, Y.J.; Ramezani, M. A fixed point theorem for quasi-contraction mappings in partially order modular spaces with an application. UPB Sci. Bull. Ser. A 2014, 76, 135-146.

21. Kim, H.-M.; Shin, H.-Y. Refined stability of additive and quadratic functional equations in modular spaces. J. Inequal. Appl. 2017, 2017, 146. [CrossRef] [PubMed]

22. Park, C.; Bodaghi, A.; Kim, S.O. A fixed point approach to stability of additive mappings in modular spaces without $\Delta_{2}$-conditions. J. Comput. Anal. Appl. 2018, 24, 1038-1048.

23. Park, C.; Rassias, J.M.; Bodaghi, A.; Kim, S.O. Approximate homomorphisms from ternary semigroups to modular spaces. Rev. Real Acad. Cienc. Exactas Fis. Nat. Ser. A-Mat. 2019, 113, 2175-2188. [CrossRef]

24. Sadeghi, G. A fixed point approach to stability of functional equations in modular spaces. Bull. Malays. Math. Sci. Soc. Second Ser. 2014, 37, 333-344.

25. Wongkum, K.; Chaipunya, P.; Kumam, P. On the generalized Ulam-Hyers-Rassias stability of quadratic mappings in modular spaces without $\Delta_{2}$-conditions. J. Funct. Spaces 2015, 2015, 461719. [CrossRef]

26. Wongkum, K.; Kumam, P.; Cho, Y.J.; Thounthong, P.; Chaipunya, P. On the generalized Ulam-Hyers-Rassias stability for quartic functional equation in modular spaces. J. Nonlinear Sci. Appl. 2017, 10, 1399-1406. [CrossRef]

27. Cădariu, L.; Radu, V. Fixed points and the stability of Jensen's functional equation. J. Inequal. Pure Appl. Math. 2003, 4, 4 .

28. Diaz, J.; Margolis, B. A fixed point theorem of the alternative for contractions on a generalized complete metric space. Bull. Amer. Math. Soc. 1968, 74, 305-309. [CrossRef]

29. Bag T.; Samanta, S.K. Finite dimensional fuzzy normed linear spaces. J. Fuzzy Math. 2003, 11, $687-705$.

30. Mirmostafaee, A.K.; Mirzavaziri, M.; Moslehian, M.S. Fuzzy stability of the Jensen functional equation. Fuzzy Sets Syst. 2008, 159, 730-738. [CrossRef]

31. Mirmostafaee, A.K.; Moslehian, M.S. Fuzzy versions of Hyers-Ulam-Rassias theorem. Fuzzy Sets Syst. 2008, 159, 720-729. [CrossRef]

32. Bînzar T.; Pater, F.; Nădăban, S. On fuzzy normed algebras. J. Nonlinear Sci. Appl. 2016, 9, 5488-5496. [CrossRef]

33. Miheț, D.; Radu, V. On the stability of the additive Cauchy functional equation in random normed spaces. J. Math. Anal. Appl. 2008, 343, 567-572. [CrossRef]

34. Bresăr, M. Jordan mappings of semiprime rings. J. Algebra 1989, 127, 218-228. [CrossRef]

(c) 2019 by the authors. Licensee MDPI, Basel, Switzerland. This article is an open access article distributed under the terms and conditions of the Creative Commons Attribution (CC BY) license (http://creativecommons.org/licenses/by/4.0/). 